Recepción: 22/11/2018

Aceptación: 10/01/2018

Publicación: 20/02/2019

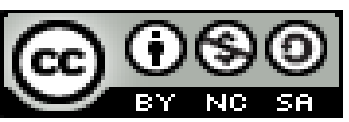

Ciencias económicas y empresariales

Artículo de investigación

\title{
Aportes teóricos a la gestión de calidad, orientados al servicio al cliente de empresas de la construcción
}

\section{Peoples and nationalities of Ecuador, a look at their presence and contribution to education and poverty in the country}

\section{Povos e nacionalidades do Equador, um olhar sobre a sua presença e contribuição para a educação e pobreza no país}

\author{
Aurora Azucena Ariza-Velasco ${ }^{\mathrm{I}}$ \\ Fausto Iván Guapi-Guamán II \\ guapi@espoch.edu.ec
}

Norma Isabel Domínguez-Gaibor III norma.dominguez@espoch.edu.ec

\section{Correspondencia: aurora.ariza@espoch.edu.ec}

I Magíster en Gerencia De Proyectos Educativos y Sociales, Especialista en Liderazgo y Gerencia, Diploma Superior en Diseño de Proyectos, Licenciada en Ciencias de la Educación Profesora de Enseñanza Media en la Especialización de Psicología Educativa y Orientación, Docente de Escuela Superior Politécnica del Chimborazo, Extensión Norte Amazónica, Chimborazo, Ecuador.

II Máster en Investigación en Pedagogía de la Cultura Física y el Deporte, Licenciado en Educación Física y Deporte, Docente de Escuela Superior Politécnica del Chimborazo, Extensión Norte Amazónica, Chimborazo, Ecuador.

III Magíster en Biología de la Conservación, Ingeniera en Ecoturismo, Guía Profesional de Turismo, Docente de Escuela Superior Politécnica del Chimborazo, Extensión Norte Amazónica, Chimborazo, Ecuador. 


\section{Resumen}

El presente artículo parte de un proyecto de investigación que se desarrolló en la ciudad de Guayaquil, Provincia del Guayas, específicamente en las firmas constructoras, el objetivo principal fue proponer un sistema de gestión de calidad orientado al servicio al cliente, para que las firmas dedicadas a las obras civiles de pequeña magnitud, pudieran ofrecer un mejor servicio. El estudio hecho permitió saber que estas empresas han visto un incremento en sus contratos en los últimos años. Por ello se realizó el estudio para conocer si existía inconformidad en los clientes que han solicitado sus servicios, además de conocer donde radica o cual es la raíz de estas presuntas inconformidades. Producto de esa experiencia se consideró importante hacer en este artículo unos aportes teóricos orientados al servicio al cliente de las empresas de construcción

Palabras clave: servicio; gestión de calidad; Cliente; empresas de la construcción; satisfacción.

\section{Summary}

This article is based on a project, research that was developed in the city of Guayaquil, Province of Guayas, specifically in the construction companies, the main objective was to propose a quality management system oriented to customer service, so that firms dedicated to civil works of small magnitude, could offer a better service. The study made it possible to know that these companies have seen an increase in their contracts in recent years. Therefore, the study was conducted to find out if there was disagreement in the clients that have requested their services, in addition to knowing where it is located or what is the root of these alleged nonconformities. As a result of this experience, it was considered important to make theoretical contributions in this article aimed at customer service of construction companies

Keywords: service; quality management; customer; construction companies; satisfaction.

\section{Resumo}

Este artigo é baseado em um projeto de pesquisa que foi desenvolvido na cidade de Guayaquil, província de Guayas, especificamente nas empresas de construção, o objetivo principal foi propor um sistema de gestão da qualidade orientada para atendimento ao cliente, para que as empresas dedicadas a obras civis de pequena magnitude, poderiam oferecer um melhor serviço. $\mathrm{O}$ estudo possibilitou saber que essas empresas registraram aumento em seus contratos nos últimos anos. 
Portanto, o estudo foi realizado para verificar se houve discordância nos clientes que solicitaram seus serviços, além de saber onde está localizado ou qual a raiz dessas supostas não conformidades. Como resultado dessa experiência, considerou-se importante fazer contribuições teóricas neste artigo visando o atendimento de empresas construtoras.

Palavras-chave: serviço; gestão de qualidade; cliente; empresas de construção civil; satisfação

\section{Introducción}

La construcción, como motor de la economía, tanto de infraestructura como de vivienda, es un sector el cual ofrece índices de crecimiento económico, en la construcción, no solamente, evidencia un estancamiento de la economía nacional, sino para la mayoría de profesionales vinculados a este sector, un sabor amargo en cuanto a su futuro profesional.

Para este trabajo, se asume que el objetivo de los profesionales de la construcción, en su mayoría, es la creación de empresas especialmente contratistas, y por falta de un desarrollo estratégico y de gestión no consiguen permanecer en el mercado ni cumplir sus objetivos de enriquecimiento y competitividad. La innovación tecnológica, la apertura de nuevos mercados, la globalización de la economía, el desarrollo tecnológico, factores políticos y regionales, son variables, a tener en cuenta, en el sector de la construcción. Y representa, entre otros, los puntos más relevantes para la sostenibilidad y creación de empresas competitivas, siendo la mano de obra no calificada es uno de los pilares más importantes para la consolidación y el crecimiento de la sinergia de las empresas de construcción de Obras Civiles.

El negocio de la construcción no debe estar dirigido sólo a la entrega de proyectos, sino, también, ofrecerle el cliente, no solamente, una construcción sino hacerle entender que el futuro depende de que tan satisfecho este con la adquisición de su vivienda, oficina o inmueble, pues es un factor fundamental en su calidad de vida. Dentro de su normal desarrollo las personas buscan tener una vivienda digna, es responsabilidad, de las empresas de construcción, crear ambientes propicios que se ajusten a las expectativas de los clientes potenciales de compra de vivienda.

Según Vavra (2003) en la actualidad, los resultados de cualquier negocio dependen del éxito que se tenga en mantener a la clientela, ya que un cliente satisfecho seguirá siendo cliente por mucho más tiempo y además hablará de forma positiva de su experiencia a otras personas. Esto es lo que

\section{4}

Pol. Con. (Edición núm. 30) Vol. 4, No 2, febrero 2019, pp. 332-346, ISSN: 2550 - 682X 
ha impulsado a los dueños de las firmas constructoras, a recurrir a estrategias de calidad que fomenten la creación de valor en su pequeña empresa.

Para Porter (2010), la experiencia se sabe que la mayor satisfacción para un cliente es recibir un producto terminado que cumpla con los más altos estándares de calidad ya que de esto dependen muchas vidas.

Kotler y Keller (2012), expresan que el concepto de calidad se centra en el cliente y añaden que una empresa ha ofrecido un producto o servicio de calidad únicamente cuando este alcanza o supera las expectativas del cliente. Los mismos autores llegan a la conclusión de que la calidad tiene un gran impacto en la satisfacción del cliente y la rentabilidad de las empresas. Ya que niveles más altos de calidad dan como resultado una mayor satisfacción de los clientes, lo que posibilita la asignación de precios más elevados a los productos o servicios que se ofrecen.

El servicio puede ser definido como beneficios intangibles los cuales consisten en actividades orientadas a individuos o a sus pertenencias. Bancos, aseguradoras, niñeras, restaurantes, son ejemplos de empresas que ofrecen servicios Ferrel \& Hartline (2012).

El manual de la calidad del servicio al cliente, sostiene que las empresas de servicio tienen un reto mucho más grande que las de producción o bienes, ya que las que producen no tienen un constante contacto con el cliente, mientras que las de servicios mantienen comunicación con el cliente de manera frecuente. A esto se debe la importancia que tienen los clientes al momento de ofrecer el servicio. El mismo manual señala que al momento de gestionar la calidad de una empresa también es un objetivo principal la reducción de sus costos. Los costos en los que se incurren también son un factor preponderante ya que no es lógico gastar en exceso cuando los réditos que está dejando cierta aplicación de calidad no compensan los mismos.

A manera de ilustración resumen de los resultados de la investigación referida.

Según los resultados obtenidos, un $69 \%$ de los clientes de la firma están satisfechos con los bienes tangibles que esta ofrece. En este factor se puede notar claramente que la sencillez y claridad de los trámites de documentación es el fuerte de la firma, mientras que se debería trabajar un poco más por mejorar la percepción que se le está dando al cliente acerca de los equipos y maquinarias utilizadas en los trabajos realizados. Esto se lo puede remediar al corto plazo con una correcta limpieza de los equipos, al mediano plazo podría superarse reparando 
equipos que tengan cierto defecto en su utilización; y, al largo plazo con la compra de nuevos equipos que permitan realizar las labores de una manera correcta y que además inspiren confianza y percepción de modernidad a los clientes.

De acuerdo al factor de responsabilidad, el $81 \%$ de los clientes de la firma consideran que ésta es responsable, siendo su punto más fuerte la atención y ayuda que brindan los empleados y la importancia que le dan a satisfacer las necesidades de los clientes.

Cuando se les consultó si la firma les comunicó oportunamente el momento de ejecución de los trabajos, aunque la mayoría contestó de manera positiva, queda una minoría por la que aún hay que mejorar, hay que tomar muy en cuenta estos datos ya que una de las premisas del servicio de la empresa es la atención personalizada.

La encuesta realizada a las Firmas Constructoras en la ciudad de Guayaquil, reveló tanto los puntos fuertes como los débiles que tienen las organización, entre los fuertes se destacaron la responsabilidad, la empatía y la seguridad que brinda la firma, mientras que como puntos débiles salieron a relucir la confiabilidad, ya que entre otras cosas, la firma no se ha caracterizado por entregar las obras a tiempo, también se anota como debilidad la parte tangible, esto se debe mayormente a la mala percepción que reciben los clientes acerca del estado de las maquinarias y equipos utilizados por la firma.

\section{Gestión de calidad}

El término gestión de calidad tiene significados específicos dentro de cada sector del negocio. Esta definición, que no apunta al aseguramiento de la buena calidad por la definición más general sino a garantizar que una organización o un producto sea consistente, tiene cuatro componentes, Planeamiento de la calidad, control de la calidad, aseguramiento de la calidad, mejoras en la calidad. La gestión de calidad se centra no solo en la calidad de un producto, servicio o la satisfacción de sus clientes, sino en los medios para obtenerla. Por lo tanto, la gestión de calidad utiliza al aseguramiento de la calidad y el control de los procesos para obtener una calidad más consistente (Kenneth, 2005).

Hablar de implementar un sistema de gestión de la calidad en una organización es una decisión estratégica que implica cambios y beneficios orientados a mejorar continuamente el desempeño 
de la empresa tomando en cuenta las necesidades de todas las partes interesadas y que demuestra la capacidad que tiene la organización para cumplir los requerimientos del cliente y las leyes que aplican. Este sistema constituye un conjunto de elementos mutuamente relacionados que interactúan para establecer la política, los objetivos de calidad y la forma de lograrlos.

El control de calidad orienta la organización al cumplimiento de los requisitos de la calidad y al cumplimiento de los objetivos, considerando el enfoque al cliente, el liderazgo y la participación del personal como algunos de los principios de la calidad. Un sistema de gestión de una organización, según la Norma ISO 9000:2000, se define como un conjunto de elementos mutuamente relacionados o que interactúan para establecer la política y los objetivos y para lograr dichos objetivos; por su parte, un sistema de gestión de la calidad es un sistema de gestión para dirigir y controlar una organización con respecto a la calidad (López, 2014).

La gestión de la calidad en los servicios por tanto es una parte fundamental en la administración de cualquier organización, su correcto manejo puede lograr una armonía entre los esfuerzos que se realizan por mantener contentos a los clientes y las ganancias que estas satisfacciones le pueden generar a la empresa. Según Cuatrecasas, L. (2011), existen ocho principios de administración de calidad que podrían ser aplicados por la alta gerencia de modo que lleven a la organización hacia una mejora constante en su desempeño: Enfoque al cliente; liderazgo; participación del personal; enfoque basado en procesos; enfoque de sistema para la gestión: mejora continua: enfoque basado en hechos para la toma de decisiones; relaciones mutuamente beneficiosas con el proveedor.

Se puede concluir diciendo, que un Sistema de Gestión de Calidad es una herramienta que le permite a cualquier organización planear, ejecutar y controlar las actividades necesarias para el desarrollo de la misión, a través de la prestación de servicios con altos estándares de calidad, los cuales son medidos a través de los indicadores de satisfacción Un Sistema de Gestión de Calidad es una herramienta que le permite a cualquier organización planear, ejecutar y controlar las actividades necesarias para el desarrollo de la misión, a través de la prestación de servicios con altos estándares de calidad, los cuales son medidos a través de los indicadores de satisfacción de los usuarios. 


\section{Servicio al cliente}

Martínez (2007), define como Servicio al cliente: conjunto de actividades interrelacionadas que ofrece una empresa con el fin de que el cliente obtenga el producto o servicio en el momento y lugar adecuado el cual satisfaga sus necesidades y/o expectativas, como consecuencia del precio, la imagen y la reputación de la empresa. Un servicio es cualquier actividad o beneficio que una parte pueda ofrecer a otra. Es esencialmente intangible y no se puede poseer. Su prestación no tiene por qué ligarse necesariamente a un producto físico.

Zeithaml (2008), el servicio al cliente es el servicio que se proporciona para apoyar el desempeño de los productos básicos de las empresas. Muy a menudo el servicio al cliente incluye responder preguntas, tomar pedidos y aspectos relacionados con facturación, manejo de reclamaciones y, quizá, mantenimiento y reparación previamente comprometidos.

Casas, Mar (2007) en términos simples los servicios son: acciones, procesos y ejecuciones pueden ser objetos tangibles que puedan verse, tocarse o sentirse, o en lugar de ello pueden ser acciones y ejecuciones intangibles. El sentido amplio de la definición de los servicios implica que la intangibilidad es un aspecto clave para determinar cuándo una oferta es o no un servicio, si bien esto es cierto también es verdad que muy pocos productos son meramente intangibles o totalmente tangibles, en cambio se puede decir que los servicios tienden a ser más intangibles que los productos manufacturados, los cuales se inclinan a ser más tangibles que los servicios, por ejemplo aun cuando la industria de la comida rápida se clasifica dentro de los servicios. También cuenta con muchos componentes tangibles, entre ellos los alimentos y el empaque. Aunque los automóviles se clasifican dentro del sector manufacturero, también proporcionan muchos intangibles como el transporte.

En la actualidad, el ofrecer servicios de calidad ha dejado de ser algo opcional para la mayoría de las industrias. El rápido ritmo del desarrollo de las tecnologías y del incremento de la competencia dificulta la obtención de ventajas competitivas estratégicas basada solamente en los productos físicos. Por otra parte, los consumidores son más demandantes, ellos no solamente esperan adquirir productos de alta calidad y excelencia si no también que los servicios que obtienen junto con estos sean de alto nivel. 
Los proveedores de servicios deben entender dos atributos de la calidad del servicio. Primero, la calidad la define el cliente, no el productor vendedor. Su peinador puede estar encantado con el trabajo que hizo con el cabello de usted, pero si usted piensa que su pelo se ve horrible entonces la calidad del servicio es mala. Segundo, los clientes evalúan la calidad del servicio comparando sus expectativas con sus percepciones de cómo se realiza este. En este proceso, no hay garantía de las expectativas serán razonables, ni seguridad alguna de que la percepción del desempeño por el cliente se basara en más que una sola experiencia.

Hayes (2004), el conocimiento de las percepciones y actitudes de los clientes con respecto a una organización comercial aumentara en gran medida sus oportunidades de tomar unas decisiones comerciales mejores, estas organizaciones conocerán las exigencias y expectativas de sus clientes y serán capaces de determinar si están cumpliendo dichas exigencias. Para poder utilizar las percepciones y actitudes de los clientes en la evaluación de la calidad de los productos y servicios, dichas percepciones y actitudes deben ser medidas de una forma fiable, si los instrumentos están mal desarrollados y representan de forma inadecuada la opinión de los clientes, las decisiones basadas en esta información pueden ser perjudiciales para el éxito de la organización.

Zeithaml (2008) plantea que los clientes perciben los servicios en términos de su calidad y del grado en que se sienten satisfechos con sus experiencias en general. Estos términos orientados hacia el cliente calidad y satisfacción han sido el centro de atención de ejecutivos e investigadores durante la última década o un poco más. Actualmente las compañías reconocen que pueden competir con mayor eficiencia cuando se distinguen así mismos por la calidad en el servicio que prestan y por el mejoramiento de la satisfacción al cliente.

Zeithaml (2008) asegura que la satisfacción es la respuesta de saciedad del cliente. Es un juicio acerca de que un rasgo del producto o servicio en sí mismo, proporciona un nivel placentero de recompensa que se relaciona con el consumo. Satisfacción es la evaluación que realiza el cliente respecto a un producto o servicio, en términos de si ese producto o servicio respondió a sus necesidades y expectativas. Se presume que al fracasar en el cumplimiento de las necesidades y las expectativas el resultado que se obtiene es la insatisfacción con dicho producto o servicio. 
Martínez (2007), define el proceso de gestión de la atención al cliente como un conjunto de actividades desarrolladas por las organizaciones con orientación al mercado, encaminadas a satisfacer las necesidades de los clientes e identificar sus expectativas actuales, que con una alta probabilidad serán sus necesidades futuras, a fin de poder satisfacerlas llegado el momento oportuno. Puede entenderse además como el hábito desarrollado y practicado por una organización para interpretar las necesidades y expectativas de sus clientes y ofrecerles, en consecuencia, un servicio accesible, adecuado, ágil, flexible, apreciable, útil, oportuno, seguro y confiable, aún bajo situaciones imprevistas o ante errores, de tal manera que el cliente se sienta comprendido, atendido y servido personalmente, con dedicación y eficacia, y sorprendido con mayor valor al esperado, proporcionando en consecuencia mayores ingresos y menores costos para la organización.

La calidad en el servicio al cliente no es un tema reciente dentro de las empresas, ya que desde siempre los clientes han exigido el mejor trato y la mejor atención al adquirir un producto o servicio, seguido de la confiabilidad, calidad, tiempos razonables, precios, y constante innovación por parte de las mismas, por lo cual cada una de las organizaciones se han visto a la tarea de buscar diversas alternativas para enriquecer dichas exigencias.

Actualmente, existe una gran y ardua competencia entre las empresas por ser el primero en obtener la atención del cliente, mismo que puede llegar a tardar un largo proceso para convertirse en un cliente principal, consistiendo en frecuentes visitas a la entidad, o bien, de varias adquisiciones del producto que se ofrece, dependiendo del giro de la empresa; pero que a su vez, puede bastar con solo un minuto de romper las políticas establecidas para la calidad en el servicio, para perder ese cliente y que este opte por ir hacia la competencia.

Por lo anterior, no se debe perder el objetivo de cumplir con la satisfacción al cliente, y cuidar celosamente de ellos, procurando mantener un juicio razonable y mostrando siempre una buena imagen de la empresa. Por los puntos planteados anteriormente, se cree que cada empresa debe darse a la tarea de conocer temas estratégicos sobre la calidad del servicio al cliente, para poder desempeñarla correctamente dentro de la misma y ampliar su cartera de clientes, gracias a la creación de una imagen más óptima y seguido del aumento de recomendaciones, desarrollando 
un ambiente más confortable tanto para sus empleados, clientes actuales y futuros clientes. (Fernández, 2014)

\section{Empresas de la construcción}

Las empresas constructoras son unidades de producción, que básicamente están integradas por el capital y el trabajo, y cuyas actividades están centradas en ofrecer un servicio en pro del bien común. En estas compañías, los beneficios son el resultado de una óptima administración, un índice de la marcha de la empresa, así como los reguladores de vida de la entidad, sin embargo, este no es el único factor que se debe resaltar o considerarse, sino también el ejercicio humano y moral, que son determinantes a largo plazo.

La meta principal de las empresas constructoras es ofrecer sus servicios conforme con lo estipulado en un proyecto y su respectivo contrato. Aunque en el transcurso de las actividades, pueden presentarse variaciones, pero estas deberán ser mínimas, basadas, acordadas y aceptadas por la compañía y el cliente. Las operaciones a ejecutarse en la obra deben haber sido explicadas detalladamente durante las fases previas a la operación: definición de proyecto y diseño.

Una construcción es la técnica o arte de fabricar edificaciones e infraestructuras y también llevar a cabo labores de rehabilitación y restauración. Para realizar todas las actividades concernientes a este ejercicio se necesitan recurrir a los servicios de entidades profesionales para su dirección y coordinación, a través de proyectos y planificación, aquí es donde entran en juego las empresas constructoras. Las constructoras disponen de la infraestructura y mano de obra pertinente para edificar cualquier tipo de construcción, sin darle importancia a la modalidad de suelo que se posea: fino o grueso, pedregoso, húmedo, arenoso, mixto, arcilloso o calizo cuando es abundante en sales. Además, cuenta con el manejo de los materiales como: cemento, varillas, tabiques entre otros.

Otra de las funciones que las empresas constructoras ejecutan, es efectuar un estudio de las condiciones del suelo a fin de determinar qué tipo de materiales utilizará para levantar la construcción. Pero, antes de comenzar con cualquier construcción la empresa deberá crear un boceto que luego se le enseñará a quien contrata los servicios para recibir su aprobación o efectuar todos los cambios convenientes para la obra. Después de que el proyecto ha sido aprobado, la constructora comenzará con la ejecución de la obra, recordando que un plan de 
construcción contempla un tiempo de entrega, considerando los inconvenientes que se pueden presentar.

\section{Conclusión}

Se tiene claro que, la construcción, como motor de la economía, tanto de infraestructura como de vivienda, es un sector el cual ofrece índices de crecimiento económico, en la construcción, no solamente, evidencia un estancamiento de la economía nacional, sino para la mayoría de profesionales vinculados a este sector, un sabor amargo en cuanto a su futuro profesional, por lo que se asume que el objetivo de los profesionales de la construcción, es la creación de empresas especialmente contratistas, pero por falta de desarrollos estratégico y de gestión no consiguen permanecer en el mercado ni cumplir sus objetivos de enriquecimiento y competitividad.

Por ello es muy importante desarrollar en estas empresas la innovación tecnológica, la apertura de nuevos mercados, la globalización de la economía, el desarrollo tecnológico, que son variables, a tener en cuenta, en el sector de la construcción, representando los puntos más relevantes para la sostenibilidad y creación de empresas competitivas, con mano de obra calificada.

Es importante tener claro que, los resultados de cualquier negocio dependen del éxito que se tenga en mantener a la clientela, ya que un cliente satisfecho seguirá siendo cliente por mucho más tiempo y además hablará de forma positiva de su experiencia a otras personas lo que debe obligar de las firmas constructoras, a recurrir a estrategias de calidad que fomenten la creación de valor en su empresa.

El concepto de calidad se centra en el cliente ya que se sabe que una empresa ha ofrecido un producto o servicio de calidad únicamente cuando este alcanza o supera las expectativas del cliente, la calidad tiene un gran impacto en la satisfacción del cliente y la rentabilidad de las empresas, ya que niveles más altos de calidad dan como resultado una mayor satisfacción de los clientes.

La investigación que dio origen a este articulo permitió conocer que, las Firmas Constructoras en la ciudad de Guayaquil, reveló tanto los puntos fuertes como los débiles que tienen las organización, entre los fuertes se destacaron la responsabilidad, la empatía y la seguridad que brinda la firma, mientras que como puntos débiles se encontró que las empresas no se han 
caracterizado por entregar las obras a tiempo, esto se debe mayormente a la mala percepción que reciben los clientes acerca del estado de las maquinarias y equipos utilizados por la firma.

Por eso hablar de implementar un sistema de gestión de la calidad organización es una decisión estratégica que implica cambios y beneficios orientados a mejorar continuamente el desempeño de la empresa tomando en cuenta las necesidades de todas las partes interesadas y que demuestra la capacidad que tiene la organización para cumplir los requerimientos del cliente y las leyes que aplican. La gestión de la calidad en los servicios por tanto es una parte fundamental en la administración de cualquier organización, su correcto manejo puede lograr una armonía entre los esfuerzos que se realizan por mantener contentos a los clientes y las ganancias que estas satisfacciones le pueden generar a la empresa.

Hablar de un Sistema de Gestión de Calidad es entenderle como una herramienta que permite a cualquier organización planear, ejecutar y controlar las actividades necesarias para el desarrollo de la misión, a través de la prestación de servicios con altos estándares de calidad, los cuales son medidos a través de los indicadores de satisfacción siendo además una herramienta que le permite a cualquier organización planear, ejecutar y controlar las actividades necesarias para el desarrollo de la misión, a través de la prestación de servicios con altos estándares de calidad, los cuales son medidos a través de los indicadores de satisfacción de los usuarios.

Hay entonces que entender el servicio al cliente como un conjunto de actividades interrelacionadas que ofrece una empresa con el fin de que el cliente obtenga el producto o servicio en el momento y lugar adecuado el cual satisfaga sus necesidades y/o expectativas, como consecuencia del precio, la imagen y la reputación de la empresa, siendo el servicio el servicio que se proporciona para apoyar el desempeño de los productos básicos de las empresas.

Por ello, el conocimiento de las percepciones y actitudes de los clientes con respecto a una organización comercial aumentara las oportunidades de tomar decisiones comerciales mejores, estas organizaciones conocerán las exigencias y expectativas de sus clientes y serán capaces de determinar si están cumpliendo dichas exigencias. Para poder utilizar las percepciones y actitudes de los clientes en la evaluación de la calidad de los productos y servicios, dichas percepciones y actitudes deben ser medidas de una forma fiable, si los instrumentos están mal desarrollados y 
representan de forma inadecuada la opinión de los clientes, las decisiones basadas en esta información pueden ser perjudiciales para el éxito de la organización.

Se puede entender también el proceso de gestión de la atención al cliente como un conjunto de actividades desarrolladas por las organizaciones con orientación al mercado, encaminadas a satisfacer las necesidades de los clientes e identificar sus expectativas actuales, que con una alta probabilidad serán sus necesidades futuras, a fin de poder satisfacerlas llegado el momento oportuno, siendo además el hábito desarrollado y practicado por una organización para interpretar las necesidades y expectativas de sus clientes y ofrecerles, en consecuencia, un servicio accesible, adecuado, ágil, flexible, apreciable, útil, oportuno, seguro y confiable, aún bajo situaciones imprevistas o ante errores, de tal manera que el cliente se sienta comprendido, atendido y servido personalmente, con dedicación y eficacia, y sorprendido con mayor valor al esperado, proporcionando en consecuencia mayores ingresos y menores costos para la organización.

Las empresas constructoras son unidades de producción, que básicamente están integradas por el capital y el trabajo, y cuyas actividades están centradas en ofrecer un servicio en pro del bien común, los beneficios son el resultado de una óptima administración, un índice de la marcha de la empresa, así como los reguladores de vida de la entidad, sin embargo, este no es el único factor que se debe resaltar o considerarse, sino también el ejercicio humano y moral, que son determinantes a largo plazo.

Por ello es necesario insistir en que, La meta principal de las empresas constructoras es ofrecer sus servicios conforme con lo estipulado en un proyecto y su respectivo contrato teniendo en cuenta que, en el transcurso de las actividades, pueden presentarse variaciones, pero estas deberán ser mínimas, basadas, acordadas y aceptadas por la compañía y el cliente.

Las constructoras deben disponer de una infraestructura y mano de obra pertinente para edificar cualquier tipo de construcción, y estar en capacidad de efectuar un estudio de las condiciones del suelo a fin de determinar qué tipo de materiales utilizará para levantar la construcción. Pero, antes de comenzar con cualquier construcción la empresa deberá crear un boceto que luego se le enseñará a quien contrata los servicios para recibir su aprobación o efectuar todos los cambios convenientes para la obra. Después de que el proyecto ha sido aprobado, la constructora 
comenzará con la ejecución de la obra, recordando que un plan de construcción contempla un tiempo de entrega, considerando los inconvenientes que se pueden presentar.

Con todo lo expuesto, se ha querido hacer un aporte a la temática de estudio, fundamentados en el trabajo de investigación ya referido. No se pretende agotar el tema, por el contrario, se insiste en la necesidad de continuar estudiando y profundizando en esta materia.

\section{Referencias Bibliográficas}

Casas, Mar (2007) Guía de atención al cliente, Cámara oficial de comercio, industria y Navegación de Cartagena. España, LOYGA, Artes Gráficas

Cuatrecasas, L. (2011). Gestión integral de la calidad (1era ed.). Recuperado de: https://books.google.com.ec. Barcelona: : Profit Editorial. .

Fernández, R. (2014). Sistemas de gestión de la calidad, ambiente y prevención de riesgos laborales. Su integración. (1era ed.). Recuperado de: https://books.google.com.ec. España: : Editorial Club Universitario.

Ferrel, O., \& Hartline, M. (2012). Estrategia de Marketing (5ta ed.). . México:: Cengage learning Editores, S.A.

Hayes, B. (2004) El marketing de servicio profesional. U.S.A.; Second.

Kotler, P., \& Keller, K. . (2012). Dirección de marketing (14ava ed.). . México:: Pearson Educación.

López, S. (2014). Implantación de un sistema de calidad. Los diferentes sistemas de calidad existentes en la organización (3era ed.). Recuperado de: https://books.google. com.ec. España: Ideaspropias Editorial.

Martínez (2007). Martínez Cortizas, D. (2013) Marketing experiencial: Un paso más allá de la buena atención al cliente. Mundiario. Recuperado el 21 de Julio, 2013 de http://mundiario.com/blog/2013/06/21/marketing-experiencial-un-pasomas-alla-de-laatencion-al-cliente

Porter, M. (2010). Ser competitivo (11ava ed.).Recuperado de: https://books.google.com.ec. Barcelona, España.: Ediciones Deusto.

Rose, Kenneth H. (julio de 2005). Project Quality Management: Why, What and How. Fort Lauderdale, Florida: J. Ross Publishing. p. 41. ISBN 1-932159-48-7 
Vavra, T. (2003). Cómo medir la satisfacción del cliente según la ISO 9001:2000 (2da ed.). Recuperado de: https://books.google.com.ec. Madrid, España.: FC Editorial.

Zeithaml (2008), Zeithaml, V. (2008) Boletín económico de información comercial española: Publicación semanal del Ministerio de Económica y Hacienda. México: Mc Graw-Hill. 